\section{Trabecular Juvenile Ossifying Fibroma: Updated Review of the Literature and Report of an Interesting Case}

\section{George Rallis ${ }^{1}$, Ourania Schoinohoriti ${ }^{1 *}$, Christos Krasada- kis $^{1}$, Christos Eftychiadis ${ }^{2}$, Nikolaos Nikitakis ${ }^{3}$ and Constan- tinos Mourouzis ${ }^{1}$}

${ }^{1}$ Department of Oral and Maxillofacial Surgery, KAT General Hospital of Attica, Athens, Greece

${ }^{2}$ Department of Anatomic Pathology, KAT General Hospital of Attica, Athens, Greece

${ }^{3}$ Department of Oral Medicine and Pathology, Dental School, National and Kapodistrian University of Athens, Athens, Greece

\begin{abstract}
Juvenile Ossifying Fibroma (JOF) represents a group of heterogenous, uncommon, benign fibro-osseous lesions that affect the craniofacial skeleton. JOF is distinguished by the young age of onset, a rather non-specific clinical and radiographic presentation and a potential locally aggressive behavior. According to the 2005 WHO classification JOF has two histological subtypes: Psammomatoid (PJOF) and Trabecular (TJOF). Although these are considered distinct clinicopathological entities, they share certain clinical and radiographic features and may similarly exhibit locally aggressive behavior, warranting radical surgical treatment and long-term follow-up.

Here presented is one case of TJOF, affecting the maxillary sinus of a 41-year-old male patient. This case is of special interest, since it represents one of the few, to our knowledge, reported cases of TJOF, located at the paranasal sinuses and the only case diagnosed at such an advanced age in the hitherto published literature. The above are corroborated though an updated review of the pertinent literature.
\end{abstract}

Keywords: Craniofacial region; Diagnosis; Trabecular Juvenile Ossifying Fibroma (TJOF); Treatment

\section{Introduction}

Fibro-osseous lesions are benign, characterized by a fibrous cellular stroma with foci of mineralization or ossification [1,2]. They are divided into 3 entities with decreasing frequency of appearance: osteoma, Fibrous Dysplasia (FD) and Ossifying Fibroma (OF); these share clinical, radiographic and histological findings but behave differently

*Corresponding author: Ourania Schoinohoriti, Department of Oral and Maxillofacial Surgery, KAT General Hospital of Attica, Athens, Greece, Tel: +30 6944676630; E-mail: our_schoinohoriti@yahoo.com

Citation: Rallis G, Schoinohoriti O, Krasadakis C, Eftychiadis C, Nikitakis N, et al. (2017) Trabecular Juvenile Ossifying Fibroma: Updated Review of the Literature and Report of an Interesting Case. J Otolaryng Head Neck Surg 3: 010.

Received: June 11, 2017; Accepted: July 13, 2017; Published: July 27, 2017
[3]. Osteoma is slow-growing and FD usually regresses spontaneously after puberty, advocating a "wait and see" policy, unless local complications occur $[4,5]$. On the contrary, especially the 'juvenile' histological subtype of may exhibit locally aggressive behavior with high recurrence risk, warranting radical surgical treatment [6]. Thus, proper initial diagnosis is mandatory for appropriate treatment [1].

OF-related nomenclature may be confusing, since various somewhat overlapping terms, including 'cementifying fibroma', 'cemento-ossifying fibroma', 'desmo-osteoblastoma', 'psammo-osteoid fibroma, 'juvenile ossifying fibroma, 'juvenile aggressive ossifying fibroma' or 'juvenile active ossifying fibroma' have been used, either synonymously or designating different histological subtypes [4]. According to the 2005 WHO classification of odontogenic tumors the term 'Ossifying Fibroma' (OF) comprises the histological variants of 'Conventional Ossifying Fibroma' (COF) and 'Juvenile Ossifying Fibroma' (JOF); the latter is further subdivided into the Trabecular (TJOF) and Psammomatoid (PJOF) subtypes $[7,8]$.

COF is mainly seen in adults ( $3^{\text {rd }}-4^{\text {th }}$ decade of life), arising from the periodontal ligament and affecting the tooth bearing areas of the jaws but rarely the nasoethmoidal complex; it is well-encapsulated, radiologically presenting as an expansile soft-tissue-mass, sharply demarcated from adjacent bone [9]. On the contrary, JOF seems a neoplastic product of the myxoid tissue precursor of cartilage and bone of paranasal sinuses; both its subtypes, JPOF and JTOF, are un-encapsulated, tend to infiltrate the adjacent bone, arise outside the tooth bearing areas of the craniofacial skeleton and usually contain multinucleated giant cells $[9,10]$. PJOF and TJOF are considered distinct clinicopathologic entities, since the former occurs predominantly in the sinonasal and orbital bones, affecting patients of a wider age range and older mean age than the latter that predominantly affects the jaws $[8,9]$. Aggressive local behavior is registered in some but not all cases of both subtypes and has been related to younger age and concurrent development of aneurysmal bone cysts [11-15].

Histologically, both subtypes have a similar stroma but TJOF is composed of densely cellular fibrous tissue with little collagen, containing thin strand-like trabeculae of fibrillary osteoid and woven bone, while PJOF is also densely cellular but contains spherical or lamellated calcifications (ossicles), the typical psammomatoid bodies $[1,6,8,9]$.

The objective of the present study is to report one case of TJOF, involving the maxillary sinus, treated in our Department. This is one of the few reported cases, affecting paranasal sinuses and the only, to our knowledge, hitherto diagnosed at the age of 41 years. An updated literature review was conducted, comprising case series and case reports, published over the last five years (from 2012 to 2017) to record the demographic, radiographic and histological features, prognosis and treatment of TJOF in the maxillofacial region.

\section{Case Report}

A 41-year-old male patient was referred to our Department with a painless progressive swelling of his left cheek since six months. Extraoral examination revealed a $2-\mathrm{cm}$ swelling of the left cheek, extending over the infraorbital foramen, just below the orbital rim. On palpation 
Citation: Rallis G, Schoinohoriti O, Krasadakis C, Eftychiadis C, Nikitakis N, et al. (2017) Trabecular Juvenile Ossifying Fibroma: Updated Review of the Literature and Report of an Interesting Case. J Otolaryng Head Neck Surg 3: 010.

the swelling was hard in consistency, with no fluctuation elicited. No paresthesia of the infraorbital nerve was registered (Figure 1). Intraorally there was no cortical expansion or apparent mucosal changes. Medical and family histories of the patient were noncontributory.

CT scans showed a $3 \times 2.5 \times 2 \mathrm{~cm}$ expansive, multilocular lesion of the left maxillary sinus, causing forward expansion of the anterior sinus wall and upper displacement of the orbital floor (Figures 2 and 3). A biopsy of the lesion was subsequently performed through a intraoral incision in the upper buccal sulcus and a Cadwell-luc approach of the maxillary sinus under general anaesthesia. The microscopic findings were consistent with the diagnosis of TJOF.

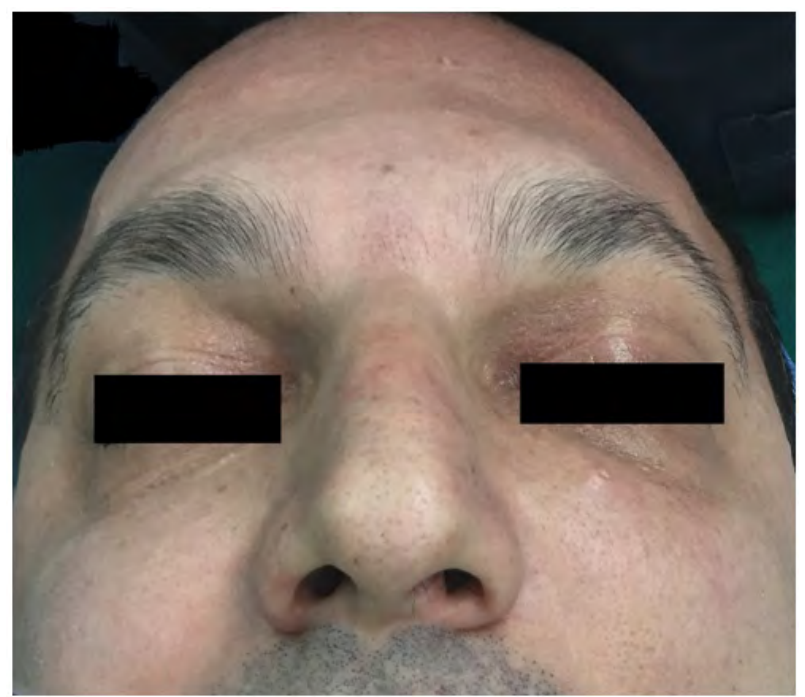

Figure 1: Original (preoperative) photograph of the patient, showing a slight swelling of his upper left cheek, over the area of the infraorbital foramen.

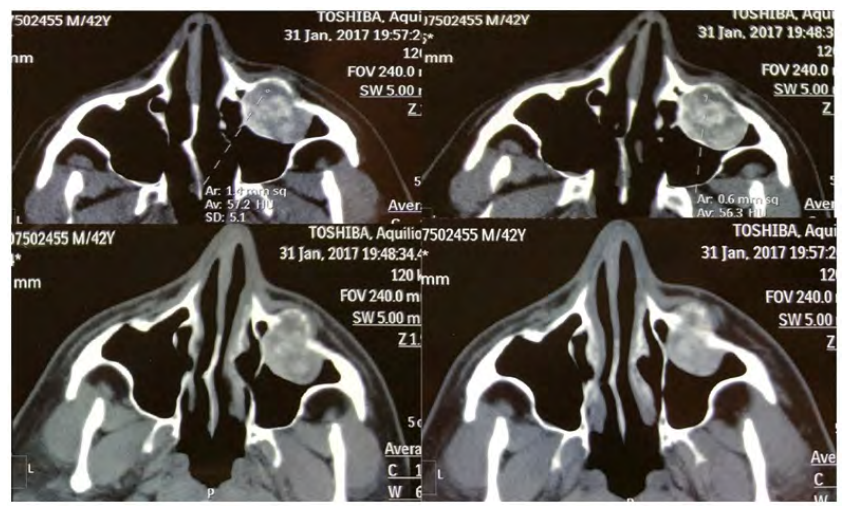

Figure 2: Preoperative axial CT views, showing an expansive lesion of the left maxillary sinus, expanding and thinning the anterior sinus wall.

A Weber-Ferguson incision was used to fully expose the lesion that occupied the upper two thirds of the maxillary sinus and infiltrated its anterior wall and the orbital floor (Figure 4). The tumor was resected en bloc within 5-mm margins, causing a considerable defect in both the orbital floor and the anterior sinus wall (Figure 5). Following resection primary reconstruction of the defects was achieved by using titanium mesh (Figure 6 and 7). The maxillary sinus was packed with antibiotic gauze for 4 days.

Histological examination of the surgical specimen confirmed the diagnosis of TJOF (Figure 8).

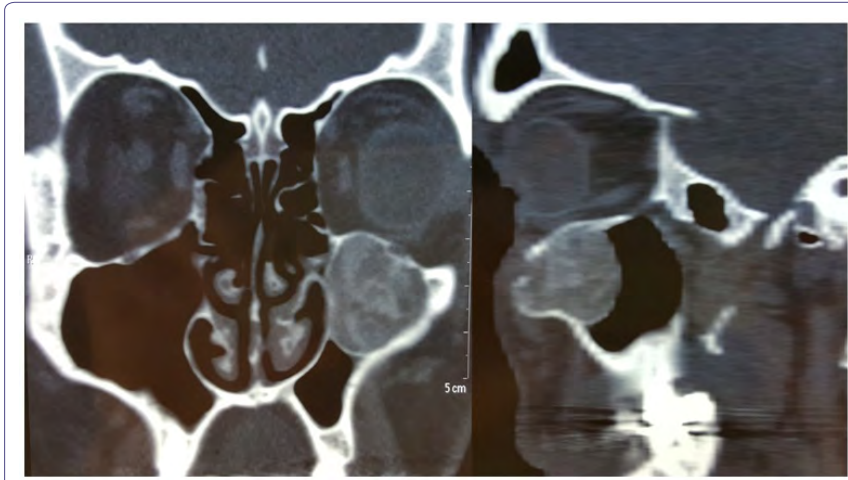

Figure 3: Preoperative coronal (left) and saggital (right) views, showing the forward expansion of the anterior sinus wall and the upper displacement of the orbital floor with infiltration of the inferior orbital rim.

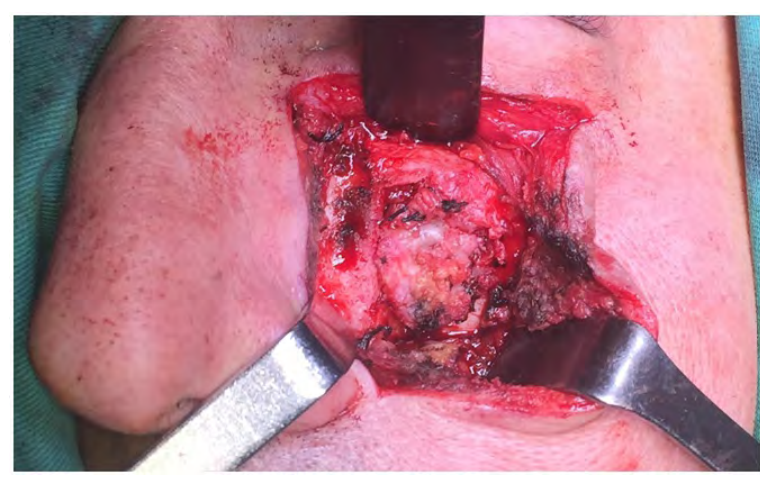

Figure 4: Intraoperative view of the tumor, occupying the upper two thirds of the maxillary sinus and infiltrating its anterior wall.

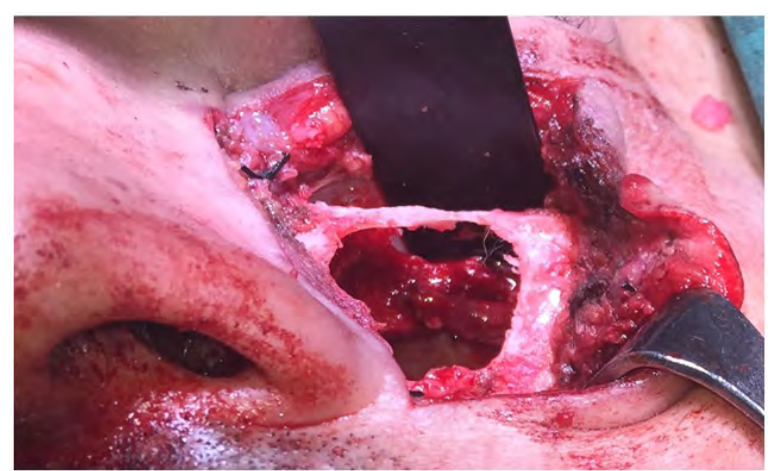

Figure 5: View of the defect at the anterior sinus wall and the orbital floor following resection of the tumor.

Facial appearance and oral function of the patient was satisfactory two months postoperatively (Figure 9); however a residual paraesthesia of the infraorbital nerve was still noticed. The patient will remain under follow-up until the case is resolved.

\section{Literature Review}

A review of the English language literature from 2012 to 2017 was conducted through the Pubmed database, to record all recently reported cases of TJOF, using the terms 'ossifying fibroma', 'juvenile ossifying fibroma', and 'trabecular juvenile ossifying fibroma'. Articles not designating the histological subtype of JOF were not included.

Our search yielded 11 articles, reporting 36 new cases of TJOF, published between 2012 and 2017 that met the inclusion criteria: 5 


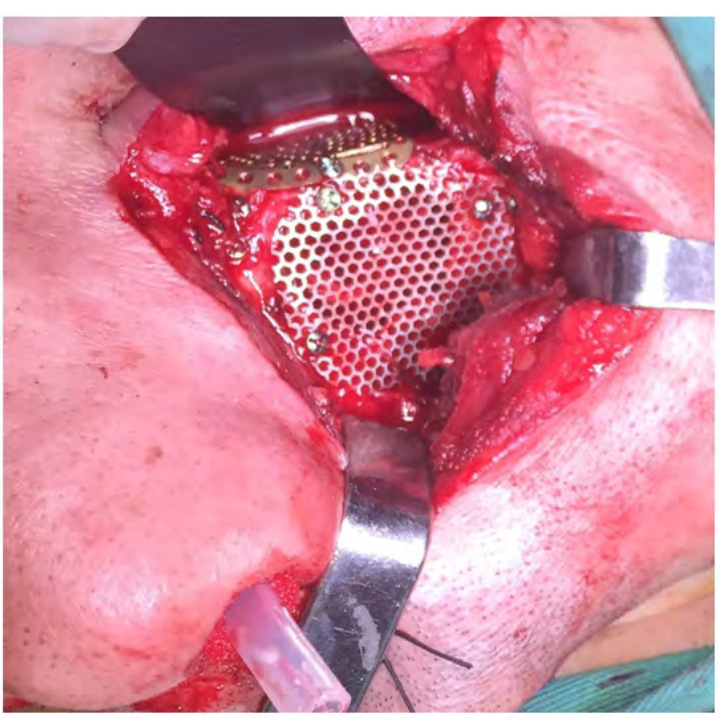

Figure 6: Primary reconstruction of the defects with the use of titanium mesh.

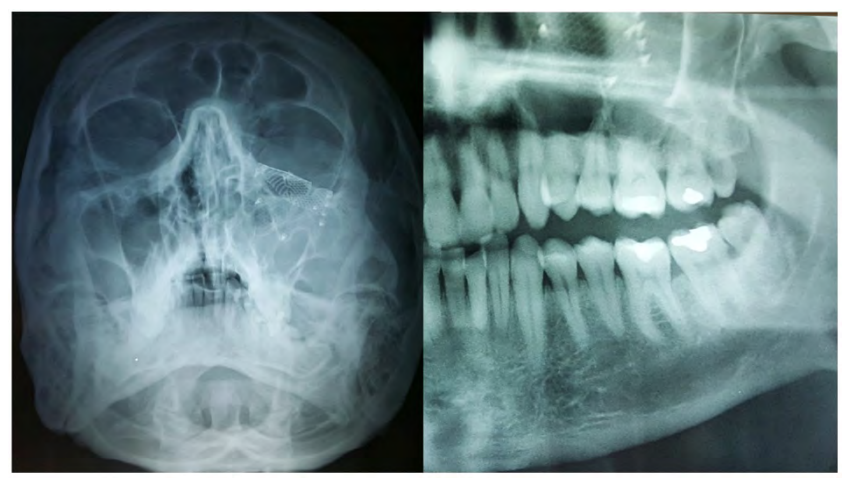

Figure 7: Postoperative radiographs-Water's (left) and orthopantomogram (right)-, showing the titanium mesh used for reconstruction of the defects.

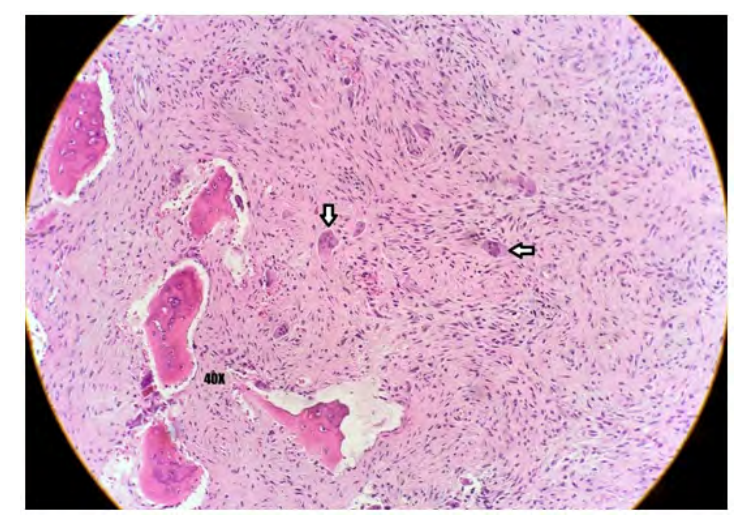

Figure 8: Photomicrographs of a hematoxylin-heosin stained section of the lesion, showing the cell-rich fibrous tissue, along with multi-nucleated giant cells (arrows) and bands of osteoid trabeculae (magnification $\times 40$ ).

case series, including cases of TJOF $[1,9,10,16,17]$ and 6 case reports [13,18-22]. Of these, 34 affected the jaws (18 the maxilla; 16 the mandible) and 2 were sinonasal (located at the maxillary sinus and nasal cavity). The age range of the patients for all 36 recently reported cases of TJOF was 3 to 25 years and the mean age was 11.8 years. The male to female ratio was 0.64: 1 (14 male and 22 female patients). Unfortunately, in the larger case series $[1,9]$ the demographic data of the

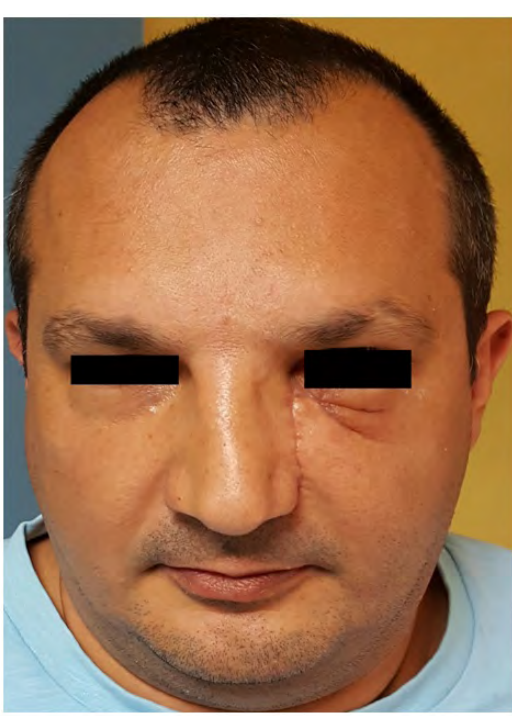

Figure 9: Photograph of the patient two months postoperatively.

patients and their histological findings were often incomplete or inconclusive. The related data are summarized in tables 1 and 2 .

\section{Discussion}

TJOF is reported less commonly and in smaller series than PJOF but may also grow rapidly [23]; it occurs within a smaller age range (233 years; mean $8.5-12$ years), shows a slight male predilection (male to female ratio 1.3:1) and affects the jaws (maxilla>mandible) $[8,18,24]$. TJOF shares clinical and radiographic findings and behaves similarly with PJOF, as far as local aggressiveness is concerned $[25,26]$. The combination of trabecular and psammomatoid patterns within the same tumor has also been reported in the literature [24].

Most TJOFs present clinically as slowly or rapidly growing masses of the craniofacial skeleton; depending on the location pain, exophthalmos, diplopia, decreasing vision, nasal congestion/obstruction, recurrent sinusitis or tooth motility/loss may appear [10,15].

Radiographically TJOF can be radiolucent, mixed or radiopaque, depending on the calcification degree and extent of cystic changes [15]. Though often expansive and destructive, it is usually well-delineated with a radiopaque border and looks like mixed or radiolucent shadows $[10,27]$. Root displacement is common but resorption rarely occurs [15].

MRI combined with CT scans help diagnosis and allows the assessment of potential involvement of adjacent structures [1]. On CT scans JOF demonstrates a dense outline, with irregular internal and smooth external surfaces and a multilocular internal appearance of varying density. MRI may show a cystic mass hypointense to muscle on T1-and T2-weighted sequences, with an outer hypo-intense shell, suggesting osteoid matrix calcification. Bony walls are iso-intense with gray on T1-and hypointense on T2-weighted images [15].

Histologically, TJOF is recognized by trabeculae of woven bone with coarse lacunae, swollen osteocytes and a lining of plump osteoblasts. Bands of cellular osteoid and hemorrhagic strands, along with multinucleated giant cells are also found. According to Slootweg a unique histological feature of TJOF are thin, garland-like, curvilinear strands of edema, hemorrhage and osteoclasts [17]. 
Citation: Rallis G, Schoinohoriti O, Krasadakis C, Eftychiadis C, Nikitakis N, et al. (2017) Trabecular Juvenile Ossifying Fibroma: Updated Review of the Literature and Report of an Interesting Case. J Otolaryng Head Neck Surg 3: 010.

- Page 4 of 5 -

\begin{tabular}{|c|c|c|c|c|c|}
\hline No. & Reference & No of TJOF/Overall of cases & Location & Mean patient age/age range (yrs) & Patient gender \\
\hline 1 & Rai et al. (2012) [16] & 2-Feb & Maxilla & 15 and 15 & 1 male +1 female \\
\hline 2 & Slootweg (2012) [17] & $15 / 15$ & 9 maxilla +6 mandible & $13.8 / 3-25$ & 6 male +9 female \\
\hline 3 & Urs et al. (2013) [9] & 22-Feb & 1 maxilla +1 mandible & 8 and 10 & 2 female \\
\hline 4 & Ciniglio Appiani et al. (2015) [1] & 11-Jan & Sinonasal & 13 & 1 female \\
\hline 5 & Han et al. (2015) [10] & 10-Oct & 4 maxilla +6 mandible & $10.9 / 7-18$ & 4 male +6 female \\
\hline
\end{tabular}

Table 1: Cases of Trabecular Juvenile Ossifying Fibroma (TJOF), included in case series of Ossifying Fibromas (OF) of the maxillofacial region, published from 2012 to 2017 (in chronological order).

(where: TJOF = Trabecular Juvenile Ossifying Fibroma; OF = Ossifying Fibroma)

\begin{tabular}{|c|c|c|c|c|}
\hline No. & Reference & Location & Patient age (yrs) & Patient gender \\
\hline 1 & B S M et al. (2012) [18] & Mandible & 7 & Male \\
\hline 2 & Osunde et al. (2013) [19] & Maxilla + maxillary sinus + nasal cavity & 7 & Female \\
\hline 3 & Pandit et al. (2014) [20] & Mandible & 13 & Male \\
\hline 4 & Reddy et al. (2014) [13] & Mandible & 16 & Female \\
\hline 5 & Rahman et al. (2015) [21] & Maxilla & 8 & Male \\
\hline 6 & Aboujaoude \& Aoun (2016) [22] & Maxilla & 7 & Female \\
\hline
\end{tabular}

Table 2: Case reports of Trabecular Juvenile Ossifying Fibroma (TJOF), published from 2012 to 2017 (in chronological order).

JOF clinical and radiographic differential diagnosis should be made from malignancies (osteosarcoma, chondrosarcoma, Ewing's sarcoma, African form of Burkitt's lymphoma, etc); however, the most important radiographic differential diagnoses are COF, FD and mixed odontogenic tumors $[15,28]$. JOF is usually monostotic and well-demarcated, lacks ground-glass attenuation and contains areas of mineralization while FD tends to be more elongated and ill-defined, demonstrating a slow growth pattern; nonetheless, polyostotic JOF, affecting both the mandible and maxilla, has been reported [29]. Mixed odontogenic tumors are well demarcated, often intimately associated with unerupted teeth and contain radiodense opacities similar to tooth structures $[15,28]$.

TJOF behaves similarly with PJOF, thus warranting the same management that remains controversial [10,30]. Locally radical surgery has been advocated to completely remove the tumor, while preserving important adjacent structures [1]. A high-up to 30-56\%-recurrence rate is mostly ascribed to incomplete excision, due to the unfavorable location and infiltrative nature of the tumor [8,27,31]. Moreover, endoscopic surgery allegedly cannot result in complete resection [32]. Therefore, in locally aggressive cases with rapid growth, cortical thinning or perforation and root displacement/resorption, total resection should be the treatment of choice $[28,33]$. However, literature lacks in data on the histological criteria, predicting biologic behavior, recurrence risk or impact of radical resection on recurrence [34,35].

Although there is no standardized follow-up protocol, long-term clinical and radiological monitoring is mandatory to detect late recurrence [27]. Immediate reconstruction is not advised because of the fairly high recurrence rate; secondary reconstruction may be undertaken sooner $(<1$ year) for slow-growing but should be delayed for fast-growing aggressive lesions [15].

The patient here presented represents the third case of TJOF, affecting paranasal sinuses, reported in the literature over the last 5 years. The first was reported in 2013 by Osunde et al., (reference \#2 of Table 2) [19] and the second in 2015 by Appiani et al., (reference \#4 of Table 1) [1]. Moreover, this case is of special interest because of the patient's age ( 41 years) that far exceeds the hitherto reported age range
(2-33 years) of patients with TJOF. Thus, this is the first, to our knowledge, TJOF case occurring at such an advanced age! Therefore, the designation 'juvenile', warranted up to now by the reported young onset age for the histological subtype of TJOF but less for the PJOF-reportedly occurring in a wider age range, up to 72 years-seems inaccurate.

\section{Conclusion}

TJOF represents one of the two histological subtypes of JOF. It is a benign but potentially locally aggressive fibro-osseous lesion, affecting mostly the jaws with an age range of 2 to 33 years. Although there are no specific guidelines to address the diagnostic and therapeutic challenges associated with TJOF, radical resection and long-term follow-up are warranted in locally aggressive cases, due to the high recurrence rate.

The here reported case of TJOF is of special interest, due to its rare location at the maxillary sinus and the advanced age of the patient (41 years) upon diagnosis. Thus, the term JOF, warranted by the hitherto reported young age of the patients, seems to be losing its foundation.

\section{References}

1. Appiani MC, Verillaud B, Bresson D, Sauvaget E, Blancal JP, et al. (2015) Ossifying fibromas of the paranasal sinuses: diagnosis and management. Acta Otorhinolaryngol Ital 35: 355-361.

2. Speight PM, Carlos R (2006) Maxillofacial fibro-osseous lesions. Current Diagnostic Pathology 12: 1-10.

3. Eller R, Sillers M (2006) Common fibro-osseous lesions of the paranasal sinuses. Otolaryngol Clin North Am 39: 585-600.

4. Ledderose GJ, Stelter K, Becker S, Leunig A (2011) Paranasal ossifying fibroma: endoscopic resection or wait and scan? Eur Arch Otorhinolaryngol 268: 999-1004.

5. Pons Y, Blancal JP, Vérillaud B, Sauvaget E, Ukkola-Pons E, et al. (2013) Ethmoid sinus osteoma: Diagnosis and management. Head Neck 35: 201204.

6. Quintero CC, Mazzei AS, Barbero JR, Diaz AP, Urzaiz LL (2016) Juvenile psammomatoid ossifying fibroma of the posterior fossa: a case report and review. Springerplus 5: 1089. 
Citation: Rallis G, Schoinohoriti O, Krasadakis C, Eftychiadis C, Nikitakis N, et al. (2017) Trabecular Juvenile Ossifying Fibroma: Updated Review of the Literature and Report of an Interesting Case. J Otolaryng Head Neck Surg 3: 010.

7. Barnes L, Eveson JW, Reichart P, Sidransky D (2005) World Health Organization Classification of Tumours. Pathology and Genetics of Head and Neck Tumours. IARC Press, Lyon, France.

8. El-Mofty S (2002) Psammomatoid and trabecular juvenile ossifying fibroma of the craniofacial skeleton: two distinct clinicopathologic entities. Oral Surg Oral Med Oral Pathol Oral Radiol Endod 93: 296-304.

9. Urs AB, Kumar P, Arora S, Augustine J (2013) Clinicopathologic and radio logic correlation of ossifying fibroma and juvenile ossifying fibroma-an institutional study of 22 cases. Ann Diagn Pathol 17: 198-203.

10. Han J, Hu L, Zhang C, Yang X, Tian Z, et al. (2016) Juvenile ossifying fibroma of the jaw: a retrospective study of 15 cases. International Journal of Oral and Maxillofacial Surgery 45: 368-376.

11. Silva CA, Silva AD, Soares JA, Furuse C, de Araújo NS, et al. (2011) Trabecular juvenile ossifying fibroma with aneurysmal bone cyst: a rare presentation. Pediatr Dent 33: 388-391.

12. Urs AB, Augustine J, Arora S, Kumar P (2013) Rare pediatric presentation of aneurysmal bone cyst with trabecular juvenile ossifying fibroma and ossifying fibroma. Int J Pediatr Otorhinolaryngol 77: 576-580.

13. Reddy AV, Reddy KR, Prakash AR, Rajinikanth, Vidhyadhari P (2014) Juvenile Ossifying Fibroma with Aneurysamal Bone Cyst: A Case Report. J Clin Diagn Res 8: 1-2.

14. Tamgadge S, Avinash T, Bhalerao S, Rajhans S (2014) Juvenile psammomatoid ossifying fibroma with aneurysmal bone cyst in the posterior mandible. Ecancermedicalscience 8: 471.

15. Sarode SC, Sarode GS, Waknis P, Patil A, Jashika M (2011) Juvenile psammomatoid ossifying fibroma: a review. Oral Oncol 47: 1110-1116.

16. Rai S, Kaur M, Goel S, Prabhat M (2012) Trabeculae type of juvenile aggressive ossifying fibroma of the maxilla: Report of two cases. Contemp Clin Dent 3: 45-50.

17. Slootweg PJ (2012) Juvenile trabecular ossifying fibroma: an update. Virchows Arch 461: 699-703.

18. Manjunatha BS, Das N, Naik S, Gowramma R (2012) Trabecular variant of juvenile aggressive ossifying fibroma of anterior mandible. Pediatr Rep 4: 24

19. Osunde O, lyogun C, Adebola R (2013) Juvenile Aggressive Ossifying Fibroma of the Maxilla: A Case Report and Review of the Literature. Ann Med Health Sci Res 3: 288-290.

20. Pandit N, Saini N, Kler S, Jindal S (2014) Juvenile trabecular ossifying fibroma. J Indian Soc Periodontol 18: 232-235.

21. Rahman T, Hashmi GS, Ansari H (2015) Trabecular Variant of Juvenile Aggressive Ossifying Fibroma. Rare Tumors 7: 5890.
22. Aboujaoude S, Aoun G (2016) Juvenile Trabecular Ossifying Fibroma of the Maxilla: a Case Report. Med Arch 70: 470-472.

23. Malathi N, Radhika T, Thamizhchelvan $\mathrm{H}$, Ravindran $\mathrm{C}$, Ramkumar $\mathrm{S}$, et al. (2011) Psammomatoid juvenile ossifying fibroma of the jaws. J Oral Maxillofac Pathol 15: 326-329.

24. Bohn OL, Kalmar JR, Allen CM, Kirsch C, Williams D, et al. (2011) Trabecular and Psammomatoid Juvenile Ossifying Fibroma of the Skull Base Mimicking Psammomatoid Meningioma. Head Neck Pathol 5: 71-75.

25. Cicciù M, Herford AS, Juodžbalys G, Cicciù D (2013) Juvenile ossifying fibroma of the maxilla: a rare aggressive case in a young patient. J Cancer Res Ther 9: 324-327.

26. Smith SF, Newman L, Walker DM, Papadopoulos H (2009) Juvenile aggressive psammomatoid ossifying fibroma: an interesting, challenging, and unusual case report and review of the literature. J Oral Maxillofac Surg 67: 200-206.

27. Tolentino ES, Centurion BS, Tjioe KC, Casaroto AR, Tobouti PL, et al. (2012) Psammomatoid juvenile ossifying fibroma: an analysis of 2 cases affecting the mandible with review of the literature. Oral Surg Oral Med Oral Pathol Oral Radiol 113: 40-45

28. Ranganath K, Kamath SM, Munoyath SK, Nandini HV (2014) Juvenile Psammomatoid Ossifying Fibroma of Maxillary Sinus: Case Report with Review of Literature. J Maxillofac Oral Surg 13: 109-114.

29. Wong WW, Motakef S, Martin MC (2016) Polyostotic Juvenile Ossifying Fibroma: An Exceptionally Rare Case. Cleft Palate Craniofac J 53: 368-372.

30. Aggarwal S, Garg A, Aggarwal A, Ahuja N, Rehman F (2012) Juvenile ossifying fibroma: Psammamatoid variant. Contemp Clin Dent 3: 330-333.

31. Wang M, Zhou B, Cui S, Li Y (2017) Juvenile psammomatoid ossifying fibroma in paranasal sinus and skull base. Acta Otolaryngol 137: 743-749.

32. Noudel R, Chauvet E, Cahn V, Mérol JC, Chays A, et al. (2009) Transcranial resection of a large sinonasal juvenile psammomatoid ossifying fibroma. Childs Nerv Syst 25: 1115-1120.

33. Abuzinada S, Alyamani A (2010) Management of juvenile ossifying fibroma in the maxilla and mandible. J Maxillofac Oral Surg 9: 91-95.

34. Colmenero-Ruiz C, Cano-Sánchez J, López-Arcas JM, Martínez-Iturriaga MT, Campo-Trapero J, et al. (2011) Multistage reconstruction in facial juvenile psammomatoid ossifying fibroma: clinical therapeutic conference. J Oral Maxillofac Surg 69: 2055-2063.

35. Juneja M, Kamboj M (2008) Juvenile ossifying fibroma of maxilla: report of a case. J Clin Pediatr Dent 33: 55-58. 